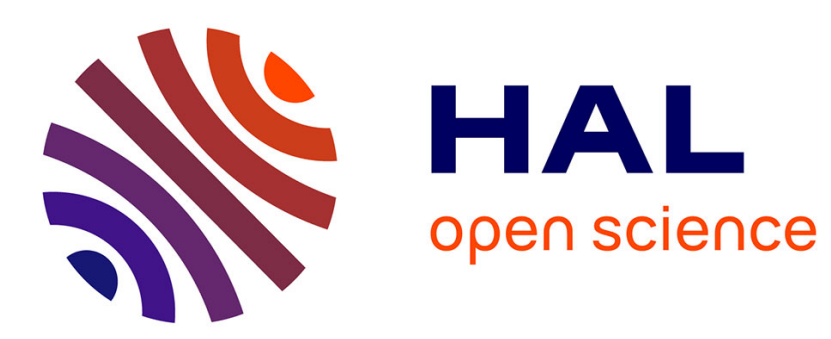

\title{
De la cybernétique aux NBIC : l'information et les machines vers le dépassement humain
}

Bernard Claverie

\section{To cite this version:}

Bernard Claverie. De la cybernétique aux NBIC : l'information et les machines vers le dépassement humain. Hermès, La Revue - Cognition, communication, politique, 2014, L'Autre n'est pas une donnée: altérité, corps et artéfacts, 68, pp.95-101. hal-01672722

\section{HAL Id: hal-01672722 \\ https://hal.science/hal-01672722}

Submitted on 4 Mar 2020

HAL is a multi-disciplinary open access archive for the deposit and dissemination of scientific research documents, whether they are published or not. The documents may come from teaching and research institutions in France or abroad, or from public or private research centers.
L'archive ouverte pluridisciplinaire HAL, est destinée au dépôt et à la diffusion de documents scientifiques de niveau recherche, publiés ou non, émanant des établissements d'enseignement et de recherche français ou étrangers, des laboratoires publics ou privés. 


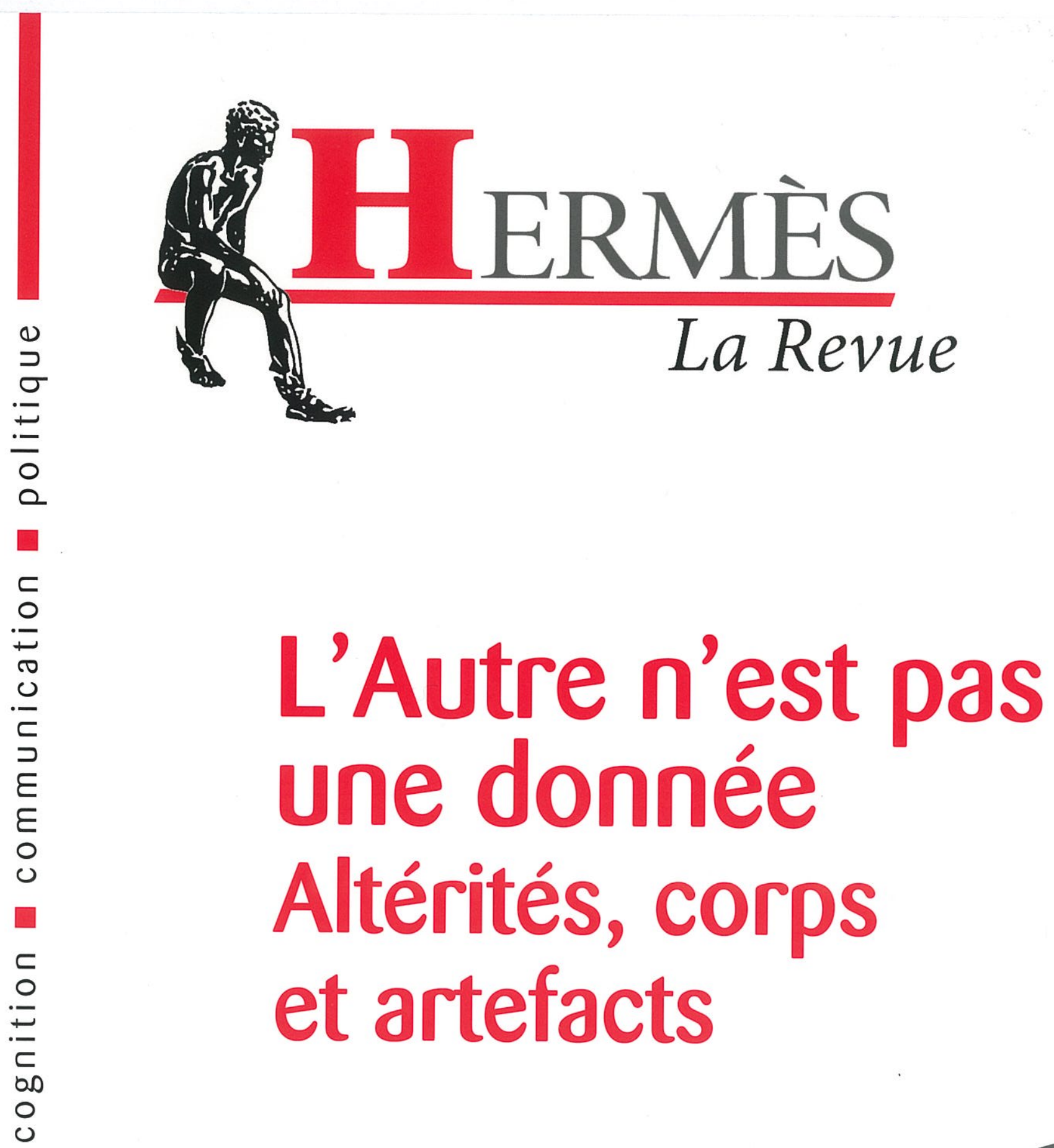




\section{Introduction}

Le vivant et l'artificiel ne se différencient plus dans les données. Apparaissent de nouveaux artefacts. La cybernétique se prolonge dans les NBIC et nos actes quotidiens se maillent toujours davantage à une couche numérique, nos relations aux faits, aux choses et à l'Autre. Ces relations sont de plus en plus liées aux algorithmes de recommandations, à une ubiquité informationnelle ou à des objets comme des lunettes hyperconnectées. L'être informationnel, quantifié, rogne avec de plus en plus de férocité l'essence, l'opacité, la transcendance de l'homme pour un humain dont les données permettraient une communication transparente, sans butée ni limite. L'Autre dans sa cognition n'est plus considéré dans toute sa particularité mais est de plus en plus perçu comme un facteur technique. Pourtant, la somme des données ne fait pas un être pensant: il y a un «truc» qui n'est pas atteint. 


\section{Bernard Claverie}

École nationale supérieure de cognitique,

Bordeaux-INP

\section{De la cybernétique aux NBIC: l'information et les machines vers le dépassement humain}

\begin{abstract}
L'émergence de la cybernétique de Wiener a donné lieu à l'un des courants de pensée des plus fertiles du $\mathrm{xx}^{\mathrm{e}}$ siècle. Dès le début, elle rencontra l'intérêt des scientifiques et des technologues. Elle fut surtout à l'origine du mouvement des technologies de l'information et de la communication. Née pendant la Seconde Guerre mondiale dans le renouveau du temps industriel porté par l'entrée des États-Unis dans le conflit mondial, la cybernétique constitua d'abord un outil conceptuel pour l'automatique, puis un immense courant de pensée dans lesquels s'inscrirent ingénieurs et électriciens, biologistes et médecins, sociologues et économistes, et nombre d'artistes tels que musiciens, plasticiens et designers. Ce bouillonnement d'idées fut, au-delà du domaine des productions technologiques, un nouveau modèle de compréhension des systèmes et de leur complexité (Lafontaine, 2004; Le Moigne, 1999; Von Bertalanffy, 1968).

Le courant trouva en France un écho particulier. L'amitié franco-américaine d'après-guerre, aussi riche que pesante, rencontrait l'aspiration des peuples à leur libération et au progrès, et les mouvements de gauche enrichis de
\end{abstract}

l'illusion sociale soviétique ambitionnaient pour l'homme une place meilleure dans le développement des systèmes technologiques, politiques et économiques. Notre pays joua alors un rôle non négligeable dans l'initiation et le développement du mouvement cybernétique, notamment avec l'électromécanicien Couffignal et sa rencontre avec l'électrophysiologiste Lapicque et le mathématicien Wiener (Aurel, 1965; Couffignal, 1958; 1972). C’est d'ailleurs à Paris que fut publié le traité fondateur de la cybernétique (Wiener, 1948).

\section{Naissance du paradigme cybernétique}

L'un des apports majeurs de la cybernétique a été de développer une théorie scientifique de l'information (Triclot, 2008). Si cette notion est aujourd'hui évidente, banalisée dans notre quotidien, il n'en était pas ainsi à l'époque. Sa généralisation au monde des machines comme à celui du vivant et des phénomènes sociaux était inattendue. Elle n'est pas étrangère au formidable mouvement 
intellectuel qu'elle engendra et généralisa, notamment autour de la conceptualisation des «machines» et des «systèmes».

Dès 1938, Couffignal définissait la machine comme « un ensemble d'êtres inanimés ou partiellement animés ou même exceptionnellement animés capables de remplacer l'homme». La conception s'ouvrait timidement à l'idée de machines naturelles, pour peu que ce soit des systèmes qui répondent à la définition d'un ensemble organisé d'éléments guidés vers un but précis. Tel fut le cas pour l'étude des grandes régulations biologiques comme l'homéostasie, et plus tard pour celle des équilibres psychologiques et même sociologiques. La notion initiale de substitution a été dépassée par Wiener lui-même, pour qui la machine est un «ensemble organisé vers un but». Les machines sont donc artificielles ou naturelles, biologiques et même sociales (Von Bertalanffy, 1968); elles deviennent des machines intelligentes (Cardon, 2004; Kurzweil, 1990), et certains veulent aujourd'hui en faire des dispositifs autonomes y compris pour leur propre réplication.

Dès les années 1950, les progrès des technologies, notamment avec le passage de la commande manuelle à l'électromécanique puis à l'électronique, ont donné naissance à deux voies complémentaires: d'une part l'automatisation des usines nouvelles puis des milieux de vie, d'autre part le calcul automatique et l'intelligence artificielle. La première voie fut celle de la complémentarité. Elle prôna l'avènement des machines automatiques, la supériorité de la robotisation et la suppression progressive des opérateurs humains. C'est d'abord pour une meilleure sécurité et une plus grande efficacité qu'elle trouva un accueil favorable dans l'industrie, puis pour une rentabilité économique s'affranchissant de l'opérateur comme élément superflu, point faible des systèmes de production.

La seconde voie fut celle du dépassement. Elle se constitue à la fois sur l'évolution de la technologie et sur l'analyse des diverses activités où l'homme peut être aidé, contrôlé voire remplacé par la machine, afin d'en éta- blir des lois de substitution orientant le programme de l'évolution technologique. Dans ce courant de pensée, la cybernétique permit l'arrivée de la nouvelle discipline informatique (mot spécifiquement francophone), à l'interface des mathématiques appliquées et de l'automatique du calcul par des machines dont la puissance en quantité et en mémoire d'informations traitées écrasait les armées de comptables ou concepteurs adeptes de la règle à calcul. Elle renvoya pour sa grande majorité le papier crayon et les tables de logarithmes au rang d'anecdotes historiques. Elle transforma la société en rendant l'homme dépendant chaque jour davantage de l'information qu'elle traite et qu'elle produit.

Substitution et dépassement: les deux concepts ont fait leur chemin. Des théoriciens s'en sont emparés, des techniciens les ont mis en œuvre, des économistes les ont édifiés comme projet de société, et des scientifiques y voient l'avenir d'une humanité débarrassée des hommes actuels, tels qu'ils sont, imparfaits.

Inspirée du mouvement cybernétique, l'idée d'une biologie moléculaire fut fondée par le physicien Weaver, juste avant qu'il ne développe avec Shannon la «théorie de l'information" (1949). Le projet du physicien était alors de transposer la rigueur et les méthodes de la physique aux sciences de la vie jusque-là réputées pour leur flou et leurs incertitudes. On notera que les premiers financements de la recherche dans le domaine furent non pas à destination des biologistes pour durcir la discipline, mais bien pour que les physiciens s'intéressent à la biologie. À la fin de la guerre, l'enjeu politique consistait aussi à inverser l'image d'une physique tournée vers la destruction, à cause de la bombe nucléaire, avec des perspectives vers la création biologique et la santé des hommes qui avaient tant souffert. Les cybernéticiens se consacraient alors aux machines intelligentes, les biologistes s'intéressaient à l'intelligence mécanisée de la vie et à l'étude de ses pierres constitutives afin de pouvoir les manipuler, réparer et créer eux-mêmes cette vie. 


\section{Vivant, matière et esprit}

Le rapprochement constructif entre physiciens et biologistes est dû à Schrödinger en 1943. Son célèbre «What is life» est imprégné des idées de la cybernétique. La notion de «code» est par exemple appliquée à la génétique et les bases conceptuelles du paradigme informationnel du vivant sont alors posées. La thermodynamique du vivant le fait apparaître non plus comme un processus permettant la reproduction, mais surtout comme un mécanisme informationnel pour échapper à l'entropie. Les biologistes adaptèrent alors la notion d'information à la compréhension des mécanismes biologiques, aux machines cybernétiques d'émergence et de maintien de la vie.

Un premier projet a été celui du décodage du langage du vivant; un second sera, au tournant du siècle, celui de sa manipulation. Le but fut d'abord de comprendre et de théoriser, puis celui d'agir. Il ambitionne aujourd'hui la réparation des erreurs de l'information biologique, la minimisation des bruits, le filtrage de l'information génétique pour en éliminer les scories ou les programmes des maladies, voire ceux responsables de la mort programmée. L'enjeu sera bientôt de permettre à l'homme une vie saine et quasi éternelle, quitte pour certain à mettre en œuvre un dépassement transhumaniste par une nouvelle espèce qui survivra à l'Homo sapiens.

Il est amusant de rappeler que, en France dans les années 1970 et 1980, les biologistes moléculaires ont tenté de s'affranchir de la cybernétique, notamment en évacuant du champ institutionnel les physiologistes intégrateurs qui se sont retrouvés cantonnés à la biologie générale des systèmes. Le mouvement systémique issu de la seconde cybernétique a fait en retour une guerre sans concessions aux réductionnistes dans l'interprétation des phénomènes de haut niveau. Qu'ils soient relatifs à l'un ou l'autre camp, les modèles ont intégré les mathématiques de la complexité dans la conception des objets d'étude, ouvrant la voie à une ingénierie du vivant.
Les deux courants - cybernétique et biologie moléculaire -, bien qu'issus d'un même élan, se sont néanmoins séparés. Ils ont été promus par des personnalités différentes qui ne se sont que rarement parlé. Les unes organisèrent leur projet de la physique vers la vie, les autres du comportement vers la modélisation et la simulation par l'artificiel. Ils se sont concurrencés, en se partageant le domaine de la modélisation de l'information, en ne se rencontrant qu'avec difficulté dans des objectifs pourtant complémentaires, les uns descendant vers la compréhension des bases de la vie, les autres remontant dans la conceptualisation des systèmes complexes de haut niveau.

Un autre courant scientifique de l'information est issu quant à lui de la physique quantique. Alors que les deux précédents s'intéressaient aux mondes macroscopique ou microscopique, des physiciens développaient des idées et des méthodes pour s'occuper des constituants invisibles de la matière: «le monde d'en bas». Ces phénomènes qui échappent à la fois à l'observation directe et aux logiques newtonienne et relativiste, sont pourtant à la base de l'organisation du monde.

Dans un discours à Caltech en 1959, le mécanicien quantique Feynman imaginait l'avenir de la recherche, celui de l'infiniment petit jusque-là quasi inexploré et surtout inexploité: "There is plenty of room at the bottom ». Le domaine à étudier est celui du tout-petit; c'est celui des briques de la matière. Il en annoncel'immensité du nombre des applications possibles, que ce soit dans les procédés d'écriture numérique, la microscopie optique, l'imagerie, la biologie moléculaire, la viscosité et la lubrification, etc. C'est en se fondant sur le monde du minuscule et des atomes qu'il imagine des technologies de cette échelle. Il appelle à ce qu'elles s'appliquent au domaine de l'information et des ordinateurs du futur, mais également à celui de la manipulation des bases biologiques du programme de la vie, de leur réparation et de leur perfectionnement. Le progrès est venu de la manipulation des atomes, un par un. Le projet est de les agencer en constructions cohérentes 
et efficaces, en constituants robustes voire indestructibles et quasi éternels, en machines qui n'ont plus rien à voir avec celles du monde sensible, en dispositifs qui traversent et s'insinuent dans les matériaux, les organes et les tissus vivants, pour s'y loger ou pour y agir en les transformant.

Les nanotechnologies correspondent aux techniques et moyens de construction et d'action à l'échelle atomique ou moléculaire, en exploitant les règles de la physique quantique. Elles constituent un nouveau domaine où l'information est conçue comme l'ensemble des propriétés d'un système de briques de base de la matière.

Les psychologues inspirés de la cybernétique se sont quant à eux différenciés selon deux axes: celui de l'inscription de la pensée dans des ensembles supérieurs, avec par exemple la psychosociologie systémique et la psycholinguistique, et celui de la psychologie expérimentale des processus cognitifs. Les sciences cognitives ont alors considéré l'appareil mental comme une machine cybernétique à traiter de l'information (Lindsay et Norman, 1977). Des neurobiologistes et psychophysiologiques, inspirés des idées néobéhavioristes (Hebb, 1949) et neurocybernétiques (Von Neumann, 1951; McCulloch, 1949), se sont inscrits dans un programme d'étude de la microstructure de la cognition (McClelland et al., 1986; Rumelhart et al., 1986). La tentative d'unification matérialiste des problématiques de l'esprit et du cerveau a été menée à partir de la combinaison des éléments cybernétiques de la pensée (Churchland, 1986).

Les sciences de la cognition s'attachent à comprendre comment s'articule, se combine et se transforme l'information pour produire de la pensée. L'essence en est l'information, le processus en est la cognition, le résultat l'intelligence et l'esprit. La tâche consiste alors à trouver les éléments actifs de traitement de l'information par des entités modulaires spécifiques, successives ou complémentaires, véritables processeurs cognitifs. De l'entrée dans le système mental, l'information est captée, décomposée, filtrée, recomposée à l'aide de gabarits perceptifs, enrichie par la mémoire, orientée par la motivation, représentée, conceptualisée, décontextualisée, prototypée, gérée par les apprentissages et des règles langagières, et organisée pour la résolution de problème et la prise de décision avant une programmation de l'action en réponse ajustée. Les mécanismes de la pensée sont autant de machines localisées que cherche aujourd'hui l'imagerie fonctionnelle avec les techniques les plus fines. La clinique neuropsychologique moderne essaye de découvrir les dysfonctionnements dans la succession des machines cognitives, des processus et de leur mise en œuvre cohérente (Loas et al., 1991; Manning, 2005).

L'analogie est posée entre le cerveau et l'ordinateur. Au-delà de la simple image d'Épinal, force est de constater la puissance de la métaphore, qu'elle soit cognitiviste ou connexionniste, dans l'évolution récente et le renouvellement des sciences cognitives. Le cerveau machine n'est pas qu'une métaphore, c'est aussi un modèle pour une meilleure compréhension de la pensée et de ses troubles (Bonnet et al., 1986; Johnson-Laird, 1988; Gardner, 1985).

\section{Vers la convergence}

Ces courants de pensée, tous inspirés de la cybernétique, se sont développés dans des secteurs scientifiques distincts. Les progrès de la technologie s'en sont inspirés, et la rationalité des technologues a poussé les chercheurs à une certaine pluridisciplinarité. On a vu ainsi des projets associant deux d'entre eux par ci, deux autres par là. Cependant, même dans cette diversité, deux notions traversent les champs concernés: l'information et les machines. Les technologues, confrontés à de vraies problématiques transdisciplinaires, ont trouvé dans ces quatre domaines la perspective d'une forte valeur ajoutée économique (Claverie, 2011). La réponse à la question ne pouvait venir que de l'interdisciplinarité en appelant à une nécessaire unification des concepts, méthodes et moyens d'action. 
La convergence est venue récemment des ÉtatsUnis. Curieusement, c'est un rapport édité à la commande conjointe de la National Science Foundation et du Department of Commerce qui a remué les deux mondes. Publié en 2003 sous l'acronyme NBIC, et sous-titré «Technologies convergentes pour améliorer les performances humaines», ce rapport fait un point de l'avancée des sciences et technologies afin d'en promouvoir la "convergence» et permettre l'accroissement des capacités et des performances des hommes grâce à des synergies entre quatre composantes applicatives NBIC. «N» pour nanotechnologies, «B» pour biotechnologies, «I» pour informatique ou technologies (cybernétiques) de l'information et $" \mathrm{C}$ » pour cognitique ou sciences cognitives appliquées. Selon les éditeurs (Roco et Bainbridge, 2003) appuyés par une cinquantaine d'experts des sciences, des humanités, de l'économie, de l'industrie ou de l'éducation, la «convergence» est la chance que se doit de saisir l'humanité. L'intégration stratégique de quatre domaines technologiques concernés doit permettre le dépassement des différences scientifiques pour un projet technologique unifié. C'est entre autres à partir de l'échelle nanométrique en remontant vers des systèmes complexes que l'on peut alors modifier, façonner, créer des machines substitutives ou d'enrichissement, voire des êtres vivants ou artificiels mais autoréplicants. Dans le premier cas, la porte est ouverte sur le transhumanisme, dans le dernier, elle s'entrebâille vers la posthumanité.

Les nanotechnologies connaissent aujourd'hui un développement tout particulier en Amérique et en Asie, avec des investissements de recherche exceptionnels et la création de nombreuses sociétés, effort qui s'est ouver plus récemment à l'Europe. Elles se spécialisent dans les nouveaux composants et le projet de nanomachines et nanorobots capables de modifier la structure moléculaire d'éléments de l'environnement ou du vivant, en agissant de l'intérieur. Les biotechnologies concernent aujourd'hui d'une part la cartographie et l'action directe sur le génome des organismes vivants, et d'autre part l'ingénierie génétique avec le séquençage et le clonage, y compris des humains. De multiples sociétés se sont développées dans le domaine depuis une dizaine d'années, orientées vers l'alimentation et l'environnement (biotechs vertes), la biologie humaine et la médecine (biotechs rouges), l'énergie ou l'industrie (biotechs blanches). Les sciences et technologies de l'information unifient informatique, électronique, automatique, télécommunications, robotique, cobotique, etc. Elles ambitionnent de futurs modes de traitemen de l'information comme l'informatique quantique ou les mondes virtuels. L'effort financier et l'intégration socioéconomique sont ici plus anciens, tant en termes d'équipements que de services. Le secteur trouve néanmoins un renouveau avec de récentes applications, l'impression $3 \mathrm{D}$ l'Internet des objets et la pervasion des composants électroniques communicants, y compris portés ou inclus au sein même d'individus devenus ainsi hybrides.

Les sciences cognitives appliquées - nommées cognitique, en France, par analogie avec le mot informatique pour en signifier l'intention technologique - fondent un projet d'étude de la pensée basé sur les sciences et technologies de l'information, les neurosciences et les sciences humaines. Discipline transverse, la cognitique associe aujourd'hui les domaines applicatifs des sciences de la communication, de l'intelligence artificielle ou hybride, des études d'usage et des réseaux, et celles des phénomènes sociaux qui les accompagnent. Elles s'inscrivent souvent dans une perspective de conception industrielle et de gestion économique, de management des connaissances ou de robustesse du traitement de l'information.

La notion centrale est celle de "convergence» Elle nécessite le bouleversement des frontières disciplinaires et le dépassement des postures des scientifiques. La convergence NBIC se fonde d'abord sur un projet d'unité matérielle, de machine à l'échelle nanométrique des objets de chacun des quatre domaines de traitement de l'information. Il s'agit de faire rencontrer le mouvement 
de la biologie, partant des êtres vivants, pour descendre vers le plus petit explicatif, celui de la cybernétique visan à mécaniser les comportements ou les substituer par des machines, celui de la cognitique pour agir sur la pensée, l'aider ou la remplacer par des mécanismes artéfactiels ou hybrides, tout ceci à partir des échelles infimes que maîtrisent les nanotechnologies. Cette révolution est aussi conceptuelle: ce n'est plus la science qui précède mais la technologie qui guide la science. Le but est l'efficacité concrète, et l'intégration est conçue à partir des plus bas niveaux pour remonter jusqu'aux objets ou aux éléments d'abord microscopiques, puis macroscopiques et même environnementaux.

Si depuis la première révolution industrielle, la science au service de la technologie et la technologie au service du pouvoir et de l'économie n'ont cessé de vouloir chaque jour encore plus dominer le monde, nous n'en sommes peutêtre avec les NBIC qu'au début d'une grande révolution conceptuelle, technique et économique. C'est en tout cas ce qu'affirment les tenants et partisans de la convergence. Mais est-ce pour le bien de l'humanité? C'est en tout cas ce que croient les fondateurs du courant NBIC, comme l'ont cru avant eux les cybernéticiens et les biologistes moléculaires, les psychologues et les informaticiens (Diamandis et Kotler, 2012; Kurzweil, 2005). Comme eux, qui ont à l'époque bénéficié de financements parfois extravagants, notamment des organismes étatiques de la défense, les technologues des NBIC développent des fondations, inspirent des universités et captent aujourd'hui des crédits

\section{RÉFÉRENCES BIBLIOGRAPHIQUES}

AUReL, D., La Cybernétique et l'humain, Paris, Gallimard, 1965.

Bonnet, C., Hoc., J.-M. et Tiberghein, G. (dir.), Psychologie, intelligence artificielle et automatique, Bruxelles, Mardaga, 1986. de recherche et de développement impressionnants. Cela témoigne de l'intérêt majeur des pays économiquement développés pour la convergence et la problématique de la mécanisation et de l'augmentation humaine.

Alors que la population, l'exploitation de ressources terminales, la pollution et le réchauffement augmentent, alors que les conflits sociaux et les guerres locales gagnent en diversité, alors que le bonheur et le vieillissement en bonne santé sont des problèmes cruciaux de nos cultures occidentales, les NBIC héritières de la cybernétique sont postulées comme l'un des enjeux prioritaires pour l'avenir de l'humanité. Mais celle-ci est alors paradoxalement conçue comme une collection de groupes de privilégiés bénéficiant de la science et de la technologie, d'individus transhumains dont on ignore ce qu'ils feront ensemble, ni même s'ils souhaiteront l'être. Cette sombre perspective est éloignée des idées humanistes des Lumières, celles d'une société égalitaire partageant des projets communs autres que celui de simplement survivre. Loin des derniers Homo sapiens et peut-être sans même la mémoire d'une altérité disparue, les NBIC ne préparent-elles pas une "humanité perdue» dont certains dénoncent aujourd'hui les dangers sans une éthique et une prise de conscience urgente (Docherty et al., 2012) ? Lavenir sera sans cela celui des post-humains; s'ils survivent, ils seront probablement dans une réalité virtuelle directement achetée au supermarché des souvenirs numériques, dans un monde de machines incontrôlées et incontrôlables, où les individus seront devenus parfaitement anecdotiques.
CARDON, A., Modéliser et concevoir une machine pensante: approche de la conscience artificielle, Paris, Vuibert, 2004 CHURChland, P. S., Neurophilosophy: Toward a Unified Science of the Mind/Brain, Cambridge, MIT Press, 1986. 
Claverie, B., Cognitique. Science et pratique des relations à la machine à penser, Paris, L'Harmattan, 2005.

Claverie, B., «Pluri-, inter-, trans-disciplinarité: ou le réel décomposé en réseaux de savoir", Projectics / Proyéctica / Projectique, $\mathrm{n}^{\circ} 4,2010$, p. 5-27.

Claverie, B., L'Homme augmenté. Néotechnologies pour un dépassement du corps et de la pensée, Paris, L'Harmattan, 2010

Couffignal, L. (dir.), Les Notions de base de la cybernétique, Paris, Gauthier-Villars, 1958.

Couffignal, L., La Cybernétique, Paris, Presses universitaires de France, coll. «Que sais-je?», 1972.

DiAmandis, P. H. et KotLeR, S., Abundance: The Future Is Better Than You Think, New York, Free Press, 2012.

DOCHERTY, B., FITZPATRICK, J., KeCK, T. et al., Losing Humanity The Case Against Killer Robots, Harvard, Human Rights Watch/ IHRC Human Rights Program, 2012.

Feyman, R., «There's Plenty of Room at the Bottom: an Invitation to Enter a New Field of Physics», conférence prononcée au congrès annuel de l'American Physical Society, California Institute of Technology, Pasadena, 29 déc. 1959, Caltech Engineering and Science, $\mathrm{n}^{\circ} 23,1960$, p. 5-22.

GARDNer, H., The Mind's New Sciences: a History of the Cognitive Revolution, New York, Basic Books, 1985.

HeвB, D. O., The Organization of Behavior, New York, John Wiley, 1949.

Johnson-Laird, P. N., The Computer and the Mind, New York Harper Collins, 1988

KurzweIL, R., The Age of Intelligent Machines, Cambridge, MIT Press, 1990.

KurzweIL, R., The Singularity is Near. When Humans Transcend Biology, New York, Penguin, 2005.

LAFONTAINE, C., L'Empire cybernétique, Paris, Seuil, 2004.

LAPICQUE, L., La Machine nerveuse, Paris, Flammarion, 1946.

LE Moigne, J.-L., La Modélisation des systèmes complexes, Paris, Dunod, 1999.

LINDSAY, P. H. et NORMAN, D., Human Information Processing, an Introduction to Psychology, New York, Academic press, 1977.
LOAS, G., Boyer, P. et SAMUel-Lajeunesse, B., Psychopathologie cognitive, Paris, Masson, 1991.

Manning, L., La Neuropsychologie clinique. Approche cognitive, Paris, Armand Colin, 2005.

MCCUlloch, W. S., «The Brain as a Computing Machine», Electrical Engineering, vol. 68, n 6, 1944, p. 492-497.

Roco, M. C. et BAINBRIDGE, W. (dir.), Converging Technologies for Improving Human Performance: Nanotechnology, Biotechnology Information Technology and Cognitive Science, Dordrecht, Kluwer, 2003.

Roco, M. C., BAinbridge, W., TonN, B. et Whitesides, G. M. (dir.), Convergence of Knowledge, Technology and Society: Beyond Convergence of Nano-bio-info-cognitive Technologies, New York, Convergence of
Springer, 2013.

Rumelhart, D. E., McClelland, J. L. et PDP Research Group (dir.), Parallel Distributed Processing. Explorations in the Microstructure of Cognition. Vol. 1: Foundations, Cambridge, MIT Press, 1986.

Rumelhart, D. E., McClelland, J. L. et PDP Research GROUP (dir), Parallel Distributed Processing Explorations in the Microstructure of Cognition. Vol. 2: Psychological and biological models, Cambridge, MIT Press, 1986

SCHrödinger, E., What is life?, Cambridge, Cambridge University Press, 1966.

Shannon, C. E., "A Mathematical Theory of Communication", Bell System Technical Journal, vol. 27, no 7, 1948, p. 379-423 et vol. $27, \mathrm{n}^{\circ} 10,1948$, p. 623-656

Triclot, M., Le Moment cybernétique, Seyssel, Champ Vallon, 2008.

VON BerTALANFFY, L., General System Theory, New York, George Braziller, 1968

Von NeumanN, J., «The General and Logical Theory of Automata", in Jefreries, L. A. (dir.) Cerebral Mechanism in Behavior. The Hixon Symposium, New York, John Wiley, 1951, p. 1-41.

Weaver, W. et Shannon, C. E., The Mathematical Theory of Communication, Urbana, University of Illinois Press, 1949.

Wiener, N., Cybernetics, or Control and Communication in the Animal and the Machine, Paris, Hermann, 1948. 